\title{
Nivolumab-induced thyroid dysfunction lacking antithyroid antibody is frequently evoked in Japanese patients with malignant melanoma
}

\author{
Seiichi Yano ${ }^{1}$, Kenji Ashida ${ }^{1 *}$ (D), Hiromi Nagata ${ }^{1}$, Kenji Ohe ${ }^{2}$, Naoko Wada $^{3}$, Yukina Takeichi ${ }^{1}$, Yuki Hanada ${ }^{1}$, \\ Yuta Ibayashi', Lixiang Wang', Shohei Sakamoto', Ryuichi Sakamoto ${ }^{1}$, Hiroshi Uchi ${ }^{3}$, Motoaki Shiratsuchi', \\ Masutaka Furue ${ }^{3}$, Masatoshi Nomura ${ }^{4}$ and Yoshihiro Ogawa ${ }^{1}$
}

\begin{abstract}
Background: Nivolumab, an anti-programmed cell death-1 monoclonal antibody, has improved the survival of patients with malignant melanoma. Despite its efficacy, nivolumab inconsistently induces thyroid dysfunction as an immune-related adverse event (irAE). This study aimed to evaluate nivolumab-induced thyroid dysfunction to determine the risks and mechanisms of thyroid irAEs.

Methods: After excluding 10 patients, data of 24 patients with malignant melanoma (aged 17-85 years; 54\% female) were retrospectively analyzed.

Results: Thyroid irAEs were observed in seven patients (29\%). Three patients had hypothyroidism after preceding transient thyrotoxicosis, and the other four patients had hypothyroidism without thyrotoxicosis. Levothyroxine-Na replacement was required in three patients. Antithyroid antibody (ATA) titer was elevated in one of four assessable patients. The average $( \pm S D)$ time to onset of thyroid irAE was $33.6( \pm 21.9)$ weeks. The administration period of nivolumab was longer in patients with thyroid irAEs than in those without thyroid irAEs $(P<0.01)$. There were no significant differences between patients with and without thyroid irAEs regarding age, sex, tumor stage, response to nivolumab therapy, baseline thyroid function, antithyroid peroxidase antibody (anti-TPO Ab) and antithyroglobulin antibody (anti-Tg Ab).

Conclusions: Thyroid dysfunction was a common irAE of nivolumab in malignant melanoma. Neither anti-TPO Ab nor anti-Tg Ab was associated with the risk for nivolumab-induced thyroid dysfunction. A conventional ATA-independent mechanism might be involved in thyroid irAEs. Further studies are required to clarify the mechanism and identify the predictive factors of thyroid irAEs.
\end{abstract}

Keywords: Thyroid, Nivolumab, Thyroiditis, Hypothyroidism, Programmed cell death -1

\section{Background}

Nivolumab, an anti-programmed cell death-1 (PD-1) antibody, has emerged as a breakthrough medication for several advanced malignancies [1, 2]; however, it frequently induces thyroid dysfunction $[3,4]$, possibly by evoked

\footnotetext{
* Correspondence: ashida@intmed3.med.kyushu-u.ac.jp

${ }^{1}$ Department of Medicine and Bioregulatory Science, Graduate School Of Medical Sciences, Kyushu University, 3-1-1 Maidashi, Higashi-Ku, Fukuoka 812-8582, Japan

Full list of author information is available at the end of the article
}

autoimmunity, similar to other immune-related adverse events (irAEs) [5-10].

Although thyroid dysfunction with thyroiditis is the most frequently reported nivolumab-induced endocrinopathy $[1,2]$, the reason why pathological autoimmunity of the thyroid is evoked by nivolumab remains unknown. In addition, autoimmune thyroid disease is common, but the mechanism of its development is not fully understood.

Here, we report the clinical characteristics and parameters of nivolumab-induced thyroid dysfunction in patients with melanoma. Thirty-four patients who were

(c) The Author(s). 2018 Open Access This article is distributed under the terms of the Creative Commons Attribution 4.0 International License (http://creativecommons.org/licenses/by/4.0/), which permits unrestricted use, distribution, and 
treated with nivolumab at our hospital were examined. This study aimed to evaluate nivolumab-induced thyroid dysfunction to determine the risks and mechanisms of thyroid irAEs.

We should take into consideration thyroid irAEs for successful treatment with this beneficial medication because we observed the occurrence of nivolumab-induced thyroid dysfunction more frequently in our study than in previous reports $[10,11]$. Furthermore, regarding nivolumab-induced thyroiditis without relation to conventional antithyroid antibody (ATA), the novel subtype of PD-1-related autoimmune thyroiditis without both antithyroid peroxidase antibody (anti-TPO Ab) and antithyroglobulin antibody (anti-Tg Ab) observed in this study might give us a novel clue in deciphering the mechanism underlying autoimmune thyroiditis.

\section{Methods}

The study retrospectively recruited all of 34 patients with advanced malignant melanoma who were treated with nivolumab (Bristol-Myers Squibb, Princeton, NJ, USA) at Kyusyu University Hospital from September 1, 2014 to September 30, 2016 (Fig. 1). The patients were intravenously administered $3 \mathrm{mg} / \mathrm{kg}$ nivolumab and evaluated thyroid function every 3 weeks. Patient characteristics such as sex, age, tumor stage, history of thyroid disorder, response to nivolumab therapy, duration of nivolumab therapy, thyroid function, and ATA were retrieved from medical records. In patients with thyroid irAEs, ATA titers were assessed at the time when thyroid dysfunction was detected (Table 1).

At the initial nivolumab therapy, 10 patients were excluded for the following reasons: six because with their thyroid function not evaluated due to early death, one with active hyperthyroidism, two with overt hypothyroidism,

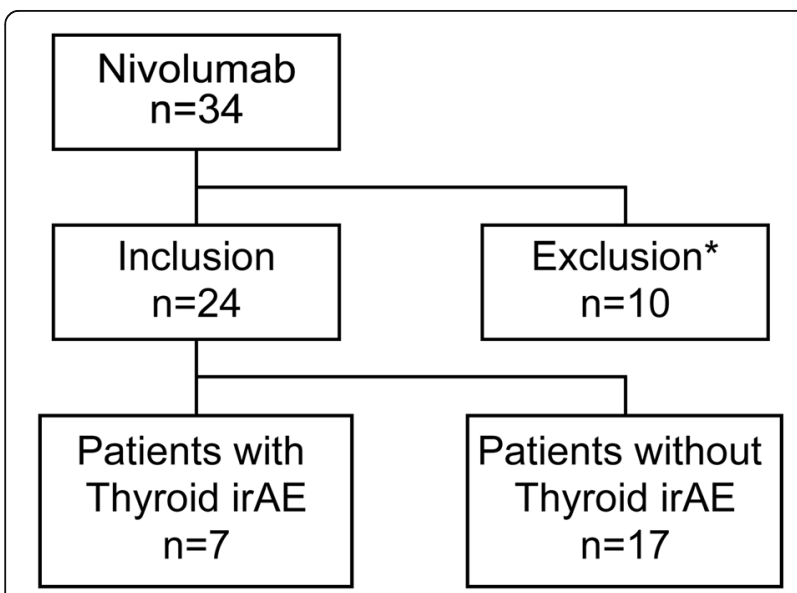

Fig. 1 Schematic overview of nivolumab-treated patients with malignant melanomaThe inclusion criteria and numbers of each group are described. irAE, immune-related adverse event. *Patients with current active thyroid dysfunction and without evaluation of thyroid function were excluded and one with secondary hypothyroidism. Two patients with a history of thyroid disorder but without ATA were included because they showed normal thyroid function and did not require active therapy: one with history of hemi-thyroidectomy due to papillary thyroid carcinoma and the other with a history of Graves' disease and having TSH receptor antibody (TRAb). None of the patients had a history of any disease that required immunomodulating agents or pretreatment with other checkpoint inhibitors such as ipilimumab, which is an anti-cytotoxic T-lymphocyte associated protein-4 antibody.

The baseline clinical features of the patients are summarized in Table 1. All the remaining 24 patients were included and evaluated for free thyroxine (fT4), free triiodothyronine (fT3), thyroid-stimulating hormone (TSH), anti-TPO Ab, and anti-Tg Ab. We defined thyrotoxicosis, hypothyroidism, and subclinical hypothyroidism according to the TSH and fT4 levels, following a previous study of irAEs [3]. Thyrotoxicosis was defined as the presence of suppressed TSH level with elevated fT4 level. Hypothyroidism was defined as the presence of elevated TSH level with decreased fT4 level. Subclinical hypothyroidism was defined as the presence of elevated TSH level with normal fT4 level. Reference range of TSH and fT4 is $0.27-4.20 \mu \mathrm{U} / \mathrm{L}$ and $1.0-1.8 \mathrm{ng} / \mathrm{dL}$, respectively. Thyroid irAEs were graded according to the Common Terminology Criteria for Adverse Events version 4.03 [12]. Baseline tumor staging was according to the American Joint Committee on Cancer (AJCC) Staging Manual, seventh edition [13].

\section{Statistical analysis}

All statistical analyses were performed using JMP ${ }^{\circledR} 13$ (SAS Institute Inc., Cary, NC, USA). Patient, tumor, and treatment variables were compared using the Fisher's exact (sex, history of previous thyroid disorder, and elevated antithyroid antibodies), Mann-Whitney-Wilcoxon (age, tumor staging, metastasis, and response to nivolumab), and the unpaired two-sample $t$ test (baseline value of mean TSH, fT4, and fT3; period of nivolumab administration; number of administration). Significance was defined as a $P$-value $<0.05$.

\section{Results}

\section{Patient characteristics}

There were no significant differences between patients with and without thyroid irAEs regarding sex, age, tumor stage, response to nivolumab therapy, and baseline thyroid function (Table 1). The average duration $( \pm \mathrm{SD})$ of nivolumab administration up to the time when thyroid irAE was diagnosed was $33.6 \pm 21.9$ weeks. The average duration of nivolumab administration was longer in patients with thyroid irAEs than in those without thyroid irAEs $(P<0.01)$. The administration durations in 
Table 1 Patient characteristics

\begin{tabular}{|c|c|c|c|c|c|}
\hline & & $\begin{array}{l}\text { Total } \\
n=24\end{array}$ & Thyroid irAE $n=7$ & $\begin{array}{l}\text { No Thyroid irAE } \\
n=17\end{array}$ & $P$ Value \\
\hline \multirow[t]{2}{*}{ Sex } & Male & 11 & 2 & 9 & \multirow[t]{2}{*}{0.39} \\
\hline & Female & 13 & 5 & 8 & \\
\hline \multirow[t]{4}{*}{ Age } & Median & $61.5 \pm 17.4$ & $57.0 \pm 19.0$ & $63.0 \pm 17.1$ & \multirow[t]{4}{*}{0.57} \\
\hline & Range & $(50-75)$ & $(37-76)$ & $(51-75)$ & \\
\hline & $\leq 40 \leq 40$ & 3 & 2 & 1 & \\
\hline & $>40$ & 21 & 5 & 16 & \\
\hline \multirow[t]{3}{*}{ Tumor staging } & $\|$ & 1 & 0 & 1 & \multirow[t]{3}{*}{0.91} \\
\hline & III & 2 & 1 & 1 & \\
\hline & IV & 21 & 6 & 15 & \\
\hline \multirow[t]{3}{*}{ Metastasis stage } & M1a & 2 & 0 & 2 & \multirow[t]{3}{*}{0.7} \\
\hline & $\mathrm{M} 1 \mathrm{~b}$ & 9 & 3 & 6 & \\
\hline & M1c & 10 & 3 & 7 & \\
\hline \multirow[t]{4}{*}{ Response to nivolumab } & $P R$ & 2 & 1 & 1 & \multirow[t]{4}{*}{0.68} \\
\hline & SD & 10 & 4 & 6 & \\
\hline & PD & 6 & 2 & 4 & \\
\hline & NA & 6 & 0 & 6 & \\
\hline \multirow[t]{2}{*}{ History of previous thyroid disorder } & YES & 2 & 1 & 1 & \multirow[t]{2}{*}{0.51} \\
\hline & NO & 22 & 6 & 16 & \\
\hline \multicolumn{2}{|l|}{ Administration duration ${ }^{\mathrm{b}}$, week } & $27.5 \pm 25.4$ & $54.4 \pm 24.1$ & $16.4 \pm 17.9$ & 0.0045 \\
\hline \multicolumn{2}{|c|}{ Administration duration including rest period ${ }^{b}$, week } & $32.1 \pm 31.4$ & $59.9 \pm 26.8$ & $20.6 \pm 26.2$ & 0.0075 \\
\hline \multicolumn{2}{|l|}{ Number of administration ${ }^{b}$} & $9.5 \pm 7.2$ & $16.7 \pm 5.4$ & $6.6 \pm 5.7$ & 0.0016 \\
\hline \multicolumn{2}{|l|}{$\mathrm{TSH}^{\mathrm{b}}, \mu \mathrm{U} / \mathrm{mL}$} & $1.94 \pm 1.30$ & $2.22 \pm 1.21$ & $1.82 \pm 1.35$ & 0.51 \\
\hline \multicolumn{2}{|l|}{$\mathrm{fT} 4^{\mathrm{b}}, \mathrm{ng} / \mathrm{dL}$} & $1.25 \pm 0.19$ & $1.19 \pm 0.13$ & $1.27 \pm 0.21$ & 0.3 \\
\hline \multicolumn{2}{|l|}{$\mathrm{fT}^{\mathrm{b}}, \mathrm{pg} / \mathrm{mL}, \mathrm{n}$} & $2.93 \pm 0.48,11$ & $3.20 \pm 0.23,3$ & $2.80 \pm 0.51,8$ & 0.063 \\
\hline \multicolumn{2}{|l|}{ Elevated anti-Tg Ab, case/total (\%) } & $2 / 17(12 \%)$ & $1 / 4(25 \%)$ & $1 / 13(8 \%)$ & 0.47 \\
\hline \multicolumn{2}{|l|}{ Elevated anti-TPO Ab, case/total (\%) } & 0/17 (0\%) & 0/4 (0\%) & 0/13 (0\%) & 1.0 \\
\hline \multicolumn{2}{|l|}{ Elevated TRAb, case/total } & $1 / 4$ & $0 / 0$ & $1 / 4$ & NA \\
\hline
\end{tabular}

Reference laboratory values are as follows: TSH, 0.27-4.20 $\mu \mathrm{U} / \mathrm{L} ;$ fT4, 1.0-1.8 ng/dL; fT3, 2.2-4.4 pg/mL; anti-TPO Ab, < 30 IU/L; anti-Tg Ab, < 30 IU/L; TRAb, <2 IU/ $\mathrm{L}$. ${ }^{2}$ Values are presented as median \pm standard deviation (SD). ${ }^{b}$ Values are presented as mean $\pm \mathrm{SD}$ and represent administration duration, including rest period; number of administrations; and TSH, fT4, and fT3 levels. Significant differences are indicated in bold font

Abbreviations: irAEs, immune-related adverse events; fT4 free thyroxine, $f T 3$ free triiodothyronine, TSH thyroid-stimulating hormone, anti-TPO Ab antithyroid peroxidase antibody, anti-Tg $A b$ antithyroglobulin antibody, TRAb TSH receptor antibody, $P R$ partial response, SD stable disease, $P D$ progressive disease; NA, not applicable

patients without thyroid irAEs were all shorter than 33.6 weeks, which was the average administration period in patients with thyroid irAEs.

\section{Thyroid-related adverse events}

Thyroid irAEs were observed in seven of 24 patients (29\%), with four having grade 1 irAEs and three having grade 2 irAEs, when we evaluated them every 3 weeks (Fig. 2). Three of the seven patients had transient thyrotoxicosis, eventually resulting in hypothyroidism. The remaining four had hypothyroidism without preceding thyrotoxicosis. Levothyroxine-Na replacement was continuously required for three of the seven patients; the thyroid function of the other four patients spontaneously resolved to normal function. Two of the three patients with overt hypothyroidism had preceding transient thyrotoxicosis.

Four patients developed secondary adrenal insufficiency, and all of them required continuous hydrocortisone replacement. One of them developed hypothyroidism 38 weeks after the start of nivolumab treatment. Another three patients developed secondary adrenal insufficiency during ipilimumab treatment after the termination of nivolumab, and two of them did not show thyroid dysfunction. The remaining patient developed hypothyroidism 5 months after the onset of secondary adrenal insufficiency, which was equivalent to 64 weeks after nivolumab administration. 


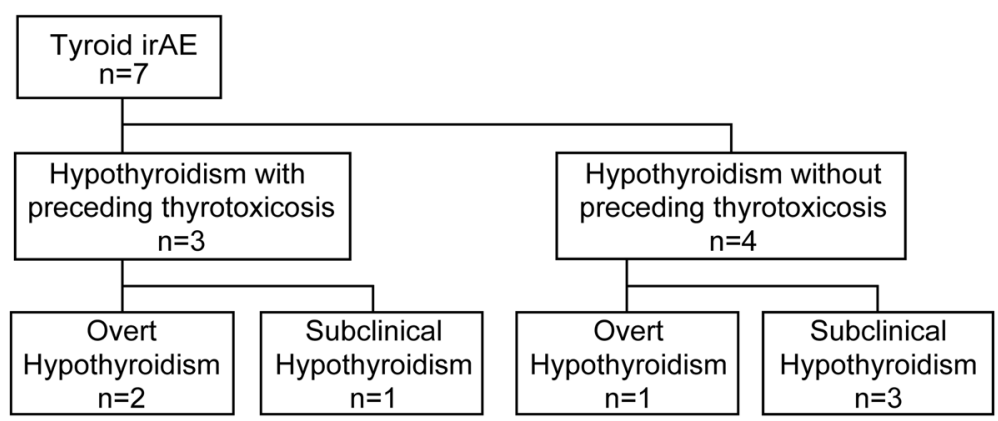

Fig. 2 Overview of thyroid immune-related adverse events (irAEs) in patients with melanoma treated with nivolumabThe continuous levothyroxine replacement was needed in two out of three hypothyroid patients who had preceding transient thyrotoxicosis and in only one out of four hypothyroid patients without preceding thyrotoxicosis.Abbreviations: irAE, immune-related adverse event

\section{Antithyroid antibodies}

ATA (anti-Tg Ab and anti-TPO Ab) titers were assessed in 17 patients (Table 1). Anti-Tg Ab titer was elevated in one of the four patients with thyroid irAEs (25\%) and one of the 13 patients without thyroid irAEs (8\%). Anti-TPO Ab was not detected in all 17 patients. TRAb titer was examined in four patients. Although one patient with a history of Graves' disease had elevated TRAb titers, other three were negative. None of the four patients developed thyroid irAEs.

\section{Discussion}

We report the clinical features of thyroid dysfunction in all patients who were treated with nivolumab for advanced malignant melanoma at our hospital. First, we found that a high proportion (29\%) of patients developed nivolumab-induced thyroid irAEs. Second, ATA titers would possibly not be useful for predicting the risk for nivolumab-induced thyroid dysfunction. Third, thyroid-specific autoimmunity induced by nivolumab might be independent of its antitumor efficacy.

A higher prevalence of thyroid dysfunction in patients with advanced melanoma treated with nivolumab was observed in this study than in previous reports. Recent pooled analysis including two phase III trials of nivolumab [10] reported $4.2 \%$ of 576 patients with melanoma complicated with hypothyroidism and $2.1 \%$ with melanoma complicated with hyperthyroidism. In another study of 206 patients with metastatic melanoma [11], nivolumab induced hypothyroidism and hyperthyroidism in $4 \%$ (nine cases) and 3\% (seven cases), respectively. In addition, $69 \%$ of the patients were from Europe or Canada and $31 \%$ were from Israel, Australia, or South America. Our study may indicate a difference in prevalence of nivolumab-induced thyroid dysfunction between Japan and Western countries. Another report of Japanese patients recently found that $21 \%$ (3/14) of nivolumab-treated patients with malignant melanoma developed thyroid dysfunction [14, 15]. Because that study did not refer to transient or subclinical hypothyroidism, the frequency of thyroid irAEs in Japan could be higher, as in our study.

Anti-TPO Ab and anti-Tg Ab titers would possibly not be useful for predicting the risk for nivolumab-induced thyroid dysfunction. In our study, only $25 \%(1 / 4)$ of patients with thyroid irAEs had elevated anti-Tg Ab titers, with none having elevated anti-TPO Ab titers (Table 1). This result suggested that at least three patients were both anti-TPO and anti-Tg Ab negative before nivolumab treatment, although anti-TPO and anti-Tg Ab have been expected to have a predictive role in thyroid irAEs $[4,14]$. Elevated ATA, especially anti-TPO $\mathrm{Ab}$ titers were shown to correlate with the development of overt hypothyroidism [16, 17]. In a previous study, anti-TPO Ab was detected in $67 \%$ of patients with painless thyroiditis after nivolumab therapy [4]. Our current study proposed that a subtype of anti-TPO and anti-Tg Ab double-negative autoimmune thyroiditis was induced by nivolumab more frequently in Japan than in Western countries. TRAb might provide us useful information on thyroid irAEs because of recent advances in TRAb titer measurement [18-20]. Although one patient with positive TRAb did not develop thyroid dysfunction, the measurement of TRAb titers might provide us valuable information on the etiology of PD-1-induced thyroid irAEs.

Thyroid-specific autoimmunity induced by nivolumab might be independent of the antitumor efficacy of nivolumab. Some studies suggest that the occurrence of irAEs in patients treated with immune checkpoint inhibitors may be correlated with an amelioration of malignancies [14, 21, 22]. Despite these reports, we found no substantial difference in antitumor effect between patients with and without thyroid irAEs, although among seven patients with thyroid irAEs, 14\% (1/7) had a clinical response compared with $6 \%(1 / 17)$ of patients without thyroid irAEs (Table 1). 
We proposed that autoimmune thyroid dysfunction without both anti-TPO and anti-Tg Ab was induced by nivolumab. Several environmental and genetic factors have been reported as triggers of autoimmune thyroid diseases [23]. The genes responsible for autoimmune thyroid diseases could be categorized as either thyroid-specific types (e.g., thyroglobulin and TSH receptor) or immune-modulating types (e.g., Forkhead box P3 (FOXP3), CD25, CD40, CTLA-4, and HLA) [23]. FOXP3 is a crucial regulatory factor for the development and function of regulatory T cells (Treg) [24], and its deficiency suppresses the regulatory function of Treg cells [25]. Furthermore, polymorphisms of FOXP3 play a role in genetic susceptibility to autoimmune thyroid diseases [26], and the genotype of FOXP3 was reported to have an association with the severity of Hashimoto's disease [27]. PD-1 is expressed on activated $\mathrm{T}$ cells, including Treg cells, and its expression by Treg cells is required to maintain immune tolerance [28]. Nivolumab-induced thyroid irAEs would clarify the etiology of conventional ATA-negative autoimmune thyroid diseases that cannot be diagnosed as definite Hashimoto's thyroiditis.

PD-1 may be a key regulator for preventing a subtype of autoimmune thyroiditis, which was found in the Japanese population. The prevalence of Hashimoto's thyroiditis correlated with the Th1 response and cellular immunity was reported to be higher in Caucasian than in Japanese, while the prevalence of Graves' disease correlated with the Th2 response was reported higher in Japanese than in Caucasians [29-31]. As the present study indicates, nivolumab-induced thyroid irAEs might be more frequently observed in Japanese, because PD-1 blockade was reported to invert the immune response from a Th2-dominant to a Th1-dominant state [31]. PD-1 blockade with nivolumab probably induced non-conventional ATA-independent autoimmunity, particularly in Th2-dominant Japanese patients.

This study has some limitations. First, the number of patients treated with nivolumab was small; thus, studies with larger number of patients will be necessary to clarify the features of nivolumab-induced thyroid irAEs. Second, we did not evaluate ATA in all patients before the onset of irAE, and the TRAb titer was not examined in patients with thyroid irAEs. Third, treatment duration, especially that in non-thyroid irAE group, was short because the occurrence of irAEs can be delayed [2]. Finally, we could not examine the histopathological features of the affected thyroid glands of patients with thyroid irAEs. Further studies are under way to identify predictive markers and to clarify the etiology of thyroid irAEs in Japanese patients. We believe these findings will uncover a subtype of autoimmune thyroid diseases without known ATA.

\section{Conclusions}

When advanced melanoma patients were treated with nivolumab, $29 \%$ of them developed thyroid dysfunction. Anti-TPO and anti-Tg Ab titers were not useful for predicting the risk for nivolumab-induced thyroid dysfunction. Therefore, constant monitoring and awareness of thyroid irAEs are recommended, particularly in patients undergoing long-term nivolumab therapy. Nivolumab may induce thyroid irAEs by evoking non-conventional thyroid-specific autoimmunity. Further studies are required to clarify the characteristics and predictive factors of thyroid irAEs.

\section{Abbreviations}

Anti-Tg Ab: antithyroglobulin antibody; Anti-TPO Ab: antithyroid peroxidase antibody; ATA: antithyroid antibody; FOXP3: Forkhead box P3; fT3: free triiodothyronine; fT4: free thyroxine; irAE: immune-related adverse event; PD-1: programmed cell death-1; TRAb: TSH receptor antibody; Treg: regulatory $T$ cells; TSH: thyroid-stimulating hormone

\section{Funding}

This research did not receive any specific grant from any funding agency in the public, commercial or not-for-profit sector.

\section{Availability of data and materials}

The datasets used and/or analyzed during the current study are available from the corresponding author on reasonable request.

\section{Authors' contributions}

SY and KA equally contributed to the study and designed the study. SY and NW collected data. SY, KA, KO, and MN analysed and interpreted data. SY and KA wrote the first draft. HN, KO, NW, YT, YH, YI, LW, SS, RS, HU, MS, MF, $\mathrm{MN}$, and $\mathrm{YO}$ provided advice and contributed to the writing of the manuscript. $S Y, K A, K O$, and MN revised the manuscript. All authors read and approved the final manuscript.

Ethics approval and consent to participate

All procedures performed in studies involving human participants were performed in accordance with the ethical standards of the Institutional Review Board of Kyushu university hospital and with the principles of the Declaration of Helsinki 2013. For this retrospective and observational study, a patient consent form was exempted by the Institutional Review Board of Kyushu university hospital. Instead, it is mandatory to disclose the research contents on website of Kyushu University Hospital. This study was approved by the Ethics Committee of Kyushu University Hospital (approval no: 29-248).

Consent for publication

Not applicable.

\section{Competing interests}

The authors declare that they have no competing interest.

\section{Publisher's Note}

Springer Nature remains neutral with regard to jurisdictional claims in published maps and institutional affiliations.

\section{Author details}

${ }^{1}$ Department of Medicine and Bioregulatory Science, Graduate School Of Medical Sciences, Kyushu University, 3-1-1 Maidashi, Higashi-Ku, Fukuoka 812-8582, Japan. ${ }^{2}$ Faculty of Pharmaceutical Sciences, Fukuoka University, 8-19-1 Nanakuma, Jonan-ku, Fukuoka 814-0180, Japan. ${ }^{3}$ Department of Dermatology, Kyushu University Hospital, 3-1-1 Maidashi, Higashi-ku, Fukuoka 812-8582, Japan. ${ }^{4}$ Division of Endocrinology and Metabolism, Department of Internal Medicine, Kurume University School of Medicine, 67 Asahi-machi, Kurume, Fukuoka 830-0011, Japan. 
Received: 26 August 2017 Accepted: 1 June 2018

Published online: 08 June 2018

\section{References}

1. Topalian SL, Hodi FS, Brahmer JR, Gettinger SN, Smith DC, McDermott DF, et al. Safety, activity, and immune correlates of anti-PD-1 antibody in cancer. N Engl J Med. 2012;366:2443-54.

2. Sznol M, Postow MA, Davies MJ, Pavlick AC, Plimack ER, Shaheen M, Veloski C, Robert C. Endocrine-related adverse events associated with immune checkpoint blockade and expert insights on their management. Cancer Treat Rev. 2017:58:70-6.

3. de Filette J, Jansen Y, Schreuer M, Everaert H, Velkeniers B, Neyns B, Bravenboer B. Incidence of thyroid-related adverse events in melanoma patients treated with pembrolizumab. J Clin Endocrinol Metab. 2016; 101:4431-9.

4. Orlov S, Salari F, Kashat L, Walfish PG. Induction of painless thyroiditis in patients receiving programmed death 1 receptor immunotherapy for metastatic malignancies. J Clin Endocrinol Metab. 2015;100:1738-41.

5. Scott LJ. Nivolumab: a review in advanced melanoma. Drugs. 2015;75: 1413-24.

6. Borghaei H, Paz-Ares L, Horn L, Spigel DR, Steins M, Ready NE, et al. Nivolumab versus docetaxel in advanced nonsquamous non-small-cell lung cancer. N Engl J Med. 2015;373:1627-39.

7. Greef B, Eisen T. Medical treatment of renal cancer: new horizons. Br J Cancer. 2016;115:505-16.

8. Okano Y, Satoh T, Horiguchi K, Toyoda M, Osaki A, Matsumoto S, et al. Nivolumab-induced hypophysitis in a patient with advanced malignant melanoma. Endocr J. 2016:63:905-12.

9. Kitajima K, Ashida K, Wada N, Suetsugu R, Takeichi Y, Sakamoto S, et al. Isolated ACTH deficiency probably induced by autoimmune-related mechanism evoked with nivolumab. Jpn J Clin Oncol. 2017:47:463-6.

10. Weber JS, Hodi FS, Wolchok JD, Topalian SL, Schadendorf D, Larkin J, et al. Safety profile of Nivolumab Monotherapy: a pooled analysis of patients with advanced melanoma. J Clin Oncol. 2017;35:785-92.

11. Robert C, Long GV, Brady B, Dutriaux C, Maio M, Mortier L, et al. Nivolumab in previously untreated melanoma without BRAF mutation. N Engl J Med. 2015:372:320-30.

12. National Cancer Institute. Common Terminology Criteria for Adverse Events (CTCAE). Version 4.03. Quick Reference. https://www.eortc.be/services/doc ctc/CTCAE_4.03_2010-06-14_QuickReference_5x7.pdf. Published June 14 (2010). Accessed 14 Aug 2017.

13. American Joint Committee on Cancer. AJCC Cancer staging manual. edn 7 ed. New York: Dordrecht, Heidelberg, London: Springer; 2010.

14. Tanaka R, Fujisawa Y, Maruyama H, Nakamura Y, Yoshino K, Ohtsuka M, Fujimoto M. Nivolumab-induced thyroid dysfunction. Jpn J Clin Oncol. 2016; 46:575-9.

15. Yamauchi I, Sakane Y, Fukuda Y, Fujii T, Taura D, Hirata M, et al. Clinical features of Nivolumab-induced thyroiditis.: a case series study. Thyroid. 2017;27:894-901.

16. Huber G, Staub JJ, Meier C, Mitrache C, Guglielmetti M, Huber P, Braverman LE. Prospective study of the spontaneous course of subclinical hypothyroidism: prognostic value of thyrotropin, thyroid reserve, and thyroid antibodies. J Clin Endocrinol Metab. 2002;87:3221-6.

17. Yoshida H, Amino N, Yagawa K, Uemura K, Satoh M, Miyai K, Kumahara Y. Association of serum antithyroid antibodies with lymphocytic infiltration of the thyroid gland: studies of seventy autopsied cases. J Clin Endocrinol Metab. 1978;46:859-62.

18. Kahaly GJ. Bioassays for TSH receptor antibodies: quo Vadis? Eur Thyroid J. 2015;4:3-5.

19. Zöphel K, Roggenbuck D, Schott M. Clinical review about TRAb assay's history. Autoimmun Rev. 2010;9:695-700.

20. McLachlan SM, Rapoport B. Thyrotropin-blocking autoantibodies and thyroid-stimulating autoantibodies: potential mechanisms involved in the pendulum swinging from hypothyroidism to hyperthyroidism or vice versa. Thyroid. 2013;23:14-24

21. Weber J, Thompson JA, Hamid O, Minor D, Amin A, Ron I, et al. A randomized, double-blind, placebo-controlled, phase II study comparing the tolerability and efficacy of ipilimumab administered with or without prophylactic budesonide in patients with unresectable stage III or IV melanoma. Clin Cancer Res. 2009; 15:5591-8.
22. Attia P, Phan GQ, Maker AV, Robinson MR, Quezado MM, Yang JC, et al. Autoimmunity correlates with tumor regression in patients with metastatic melanoma treated with anti-cytotoxic T-lymphocyte antigen-4. J Clin Oncol. 2005:23:6043-53.

23. Lee HJ, Li CW, Hammerstad SS, Stefan M, Tomer Y. Immunogenetics of autoimmune thyroid diseases: a comprehensive review. J Autoimmun. 2015; 64:82-90.

24. Yagi H, Nomura T, Nakamura K, Yamazaki S, Kitawaki T, Hori S, et al. Crucial role of FOXP3 in the development and function of human CD25+CD4+ regulatory T cells. Int Immunol. 2004;16:1643-56.

25. Okumura A, Ishikawa T, Sato S, Yamauchi T, Oshima H, Ohashi T, et al. Deficiency of forkhead box P3 and cytotoxic T-lymphocyte-associated antigen-4 gene expressions and impaired suppressor function of CD4(+) CD25(+) T cells in patients with autoimmune hepatitis. Hepatol Res. 2008;3: 896-903.

26. Ban Y, Tozaki T, Tobe T, Ban Y, Jacobson EM, Concepcion ES, Tomer Y. The regulatory T cell gene FOXP3 and genetic susceptibility to thyroid autoimmunity: an association analysis in Caucasian and Japanese cohorts. J Autoimmun. 2007;28:201-7.

27. Inoue N, Watanabe M, Morita M, Tomizawa R, Akamizu T, Hidaka Y, Iwatan $Y$. Association of functional polymorphisms related to the transcriptional level of FOXP3 with prognosis of autoimmune thyroid diseases. Clin Exp Immunol. 2010;162:402-6.

28. Zhang B, Chikuma S, Hori S, Fagarasan S, Honjo T. Nonoverlapping roles of PD-1 and FoxP3 in maintaining immune tolerance in a novel autoimmune pancreatitis mouse model. Proc Natl Acad Sci U S A. 2016;113:8490-5.

29. McLeod DS, Caturegli P, Cooper DS, Matos PG, Hutfless S. Variation in rates of autoimmune thyroid disease by race/ethnicity in US military personnel. JAMA. 2014;311:1563-5.

30. Phenekos C, Vryonidou A, Gritzapis AD, Baxevanis CN, Goula M, Papamichail M. Th1 and Th2 serum cytokine profiles characterize patients with Hashimoto's thyroiditis (Th1) and Graves' disease (Th2). Neuroimmunomodulation. 2004;11: 209-13

31. Dulos J, Carven GJ, van Boxtel SJ, Evers S, Driessen-Engels LJ, Hobo W, et al. PD-1 blockade augments Th1 and Th17 and suppresses Th2 responses in peripheral blood from patients with prostate and advanced melanoma cancer. J Immunother. 2012;35:169-78.

\section{Ready to submit your research? Choose BMC and benefit from:}

- fast, convenient online submission

- thorough peer review by experienced researchers in your field

- rapid publication on acceptance

- support for research data, including large and complex data types

- gold Open Access which fosters wider collaboration and increased citations

- maximum visibility for your research: over $100 \mathrm{M}$ website views per year

At BMC, research is always in progress.

Learn more biomedcentral.com/submissions 